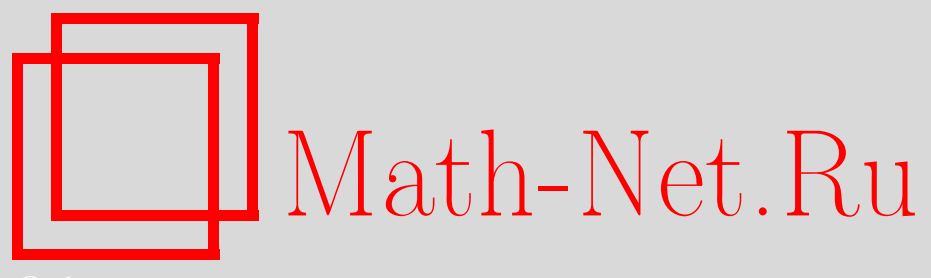

Д. Д. Киселев, Об оценке индекса Шура неприводимых представлений конечных групп, Матем. сб., 2013, том 204, номер 8, 73-82

DOI: https://doi.org/10.4213/sm8175

Использование Общероссийского математического портала Math-Net.Ru подразумевает, что вы прочитали и согласны с пользовательским соглашением http://www . mathnet.ru/rus/agreement

Параметры загрузки:

IP: 3.85 .5 .30

26 апреля 2023 г., 17:30:14

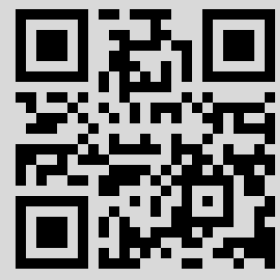




\title{
Д. Д. Киселев \\ Об оценке индекса Шура неприводимых представлений конечных групп
}

\begin{abstract}
Строится оптимальная оценка индекса Шура над полем рациональных чисел неприводимых комплексных представлений конечных групп, если известны лишь простые делители порядка группы. Изучаются взаимосвязи с согласными и универсально согласными расширениями числовых полей. Приводится более простое доказательство известной оценки Бермана-Ямады индекса Шура над полем $\mathbb{Q}_{p}$.

Библиография: 7 названий.
\end{abstract}

Ключевые слова: конечная группа, индекс Шура, универсально согласные расширения.

DOI: $10.4213 / \operatorname{sm} 8175$

\section{$\S 1$. Введение}

1.1. В теории конечных групп наиболее важными с точки зрения связей с другими разделами алгебры частями являются теория представлений и теория Галуа (обратная задача). Их взаимное влияние друг на друга хорошо иллюстрируется с помощью теории индекса Шура с одной стороны и теории задач погружения с другой. В самом деле, изучение оценок индекса Шура неприводимого характера $\chi$ конечной группы $G$ над некоторым полем нулевой характеристики $k$ сводится к изучению индекса соответствующей центрально-простой компоненты $A(k(\chi), \chi)$ групповой алгебры $k(\chi) G$ (см., например, [1]). Согласно фундаментальной теореме Брауэра-Витта (см. [1; гл. 3, теорема Брауэра-Витта]) изучение алгебры $A(k(\chi), \chi)$ сводится к изучению эквивалентной ей в смысле группы Брауэра $B(k(\chi))$ циклотомической алгебре

$$
\left(k\left(\varepsilon_{\exp G}\right) / k(\chi), f\right),
$$

где $f$ - 2-коцикл группы $Z^{2}\left(\operatorname{Gal}\left(k\left(\varepsilon_{\exp G}\right) / k(\chi)\right),\left\langle\varepsilon_{\exp G}\right\rangle\right)$.

$\mathrm{C}$ другой стороны, в теории задач погружения (см. [2]) ставится следующая проблема: рассматривается расширение конечных групп

$$
1 \longrightarrow N \longrightarrow H \stackrel{\varphi}{\longrightarrow} \operatorname{Gal}(K / k) \longrightarrow 1
$$

требуется так построить алгебру Галуа $L$ с группой $k$-автоморфизмов $H$, содержащую поле $K$, чтобы эпиморфизм ограничения $k$-автоморфизмов $L$ на поле $K$ совпадал с эпиморфизмом $\varphi$. Данная задача полностью решена для случая абелева ядра $N$ А. В. Яковлевым в работе [3]. Существует важное необходимое 
условие разрешимости задачи погружения $(K / k, H, \varphi)$, называемое условием согласности Д. К. Фаддеева-Х. Хассе (см. [2; гл. 2]). Условие согласности для данной задачи для случая абелева ядра $N$ состоит в следующем. Будем предполагать, что $\varepsilon_{\exp N} \in K$. Это допущение, как показано в [2; теорема 2.6], не ограничивает общность. Пусть $H_{1}$ - подгруппа в $H$, содержащая ядро $N$. Пусть далее $k_{1}$ - подполе $K$, принадлежащее группе $\varphi\left(H_{1}\right)$, а $B$ - нормальный делитель в $H_{1}$, содержащийся в $N$. В этом случае эпиморфизм $\varphi$ индуцирует эпиморфизм $\varphi_{1}: H_{1} / B \rightarrow \varphi\left(H_{1}\right)$. Задача погружения $\left(K / k_{1}, H_{1} / B, \varphi_{1}\right)$ называется сопутствующей для задачи $(K / k, H, \varphi)$.

Задача погружения $\left(K_{1} / k_{0}, H_{2}, \beta\right)$ называется по определению брауэровской, если $\operatorname{ker} \beta$ - циклическая группа, причем существует операторный (относительно группы $\left.\operatorname{Gal}\left(K_{1} / k_{0}\right)\right)$ мономорфизм группы $\operatorname{ker} \beta$ в $K_{1}^{*}$.

Условие согласности для задачи погружения $(K / k, H, \varphi)$ состоит в разрешимости всех сопутствующих брауэровских задач.

Из результатов книги [2] следует, что расщепление циклотомической алгебры (1) происходит тогда и только тогда, когда для брауэровской задачи погружения $\left(k\left(\varepsilon_{\exp G}\right) / k(\chi), H, \varphi,\left\langle\varepsilon_{\exp G}\right\rangle\right)$ выполнено условие согласности, которое в данном случае равносильно разрешимости последней.

1.2. В п. 1.1 мы увидели возникающие взаимосвязи между теорией индекса Шура и задачами погружения. Такие взаимосвязи позволяют дать чуть более простое доказательство известной оценки Бермана-Ямады.

Теорема 1 (С.Д. Берман, Т. Ямада). Пусть $G$ - конечная группа, $\chi \in$ $\operatorname{Irr} G$. Тогда $m_{\mathbb{Q}_{p}}(\chi) \mid(p-1)$ при $p>2, m_{\mathbb{Q}_{2}}(\chi) \mid 2$.

В то же время результаты теории индекса Шура влияют на задачи погружения. В работе [4] введено понятие универсально согласного расширения. Рассмотрим конечное расширение Галуа $K / k$ и предположим, что $\varepsilon_{n} \in K$. Такое расширение называется универсально согласным периода $n$, если для любой подгруппы $F_{1}$ группы $\operatorname{Gal}(K / k)$ гомоморфизм

$$
H^{2}\left(F_{1},\left\langle\varepsilon_{n}\right\rangle\right) \rightarrow H^{2}\left(F_{1}, K^{*}\right),
$$

индуцированный вложением $\left\langle\varepsilon_{n}\right\rangle \hookrightarrow K^{*}$, является нулевым. В [4] показана исключительная важность универсально согласных расширений для обратной задачи теории Галуа. Там же (см. [4; комментарий к теореме 6]) для случая числовых полей $K / k$ показано, что проверка универсальной согласности редуцируется к проверке универсальной согласности пополненного расширения $K_{\mathfrak{p}} / k_{\mathfrak{p}}$ по некоторой простой (не обязательно неархимедовой) точке $\mathfrak{p}$ поля $k$. В пополненном же расширении указанная проверка редуцируется к проверке универсальной согласности $q$-примарного периода $q$-силовского подрасширения в расширении $K_{\mathfrak{p}} / k_{\mathfrak{p}}$ (см. [4; теорема 4]). Получить же общий критерий универсальной согласности $p$-примарного периода для $p$-расширения $p$-локальных полей $K_{\mathfrak{p}} / k_{\mathfrak{p}}$ весьма непросто. Это связано со сложным строением группы Галуа максимального $p$-расширения поля $k_{\mathfrak{p}}$. Теория индекса Шура позволяет установить следующий результат. 
Теорема 2. Рассмотрим р-расширение р-локальных полей $k\left(\varepsilon_{n}\right) / k$. Если $p=2$, то потребуем $\sqrt{-1} \in k$. Расширение $k\left(\varepsilon_{n}\right) / k$ является универсально согласным периода $n$.

\section{§ 2. Формулировка результатов}

2.1. Обозначения. Всюду далее под числовым полем будем понимать поле алгебраических чисел, под локальным полем - пополнение числового поля по некоторой простой неархимедовой точке. Если такая точка лежит над простым числом $p$, то соответствующее поле будем называть $p$-локальным. Введем для удобства следующие обозначения: $\langle X\rangle$ - группа, порожденная множеством $X$, $\operatorname{Irr} G$ - множество неприводимых комплексных характеров конечной группы $G$, $m_{k}(\chi)$ - индекс Шура неприводимого комплексного характера $\chi$ конечной группы $G$ над полем нулевой характеристики $k, A(k, \chi)$ - простая компонента групповой алгебры $k G$ конечной группы $G$, соответствующая характеру $\chi \in \operatorname{Irr} G$, поле $k$ имеет нулевую характеристику, $k_{\mathfrak{p}}$ - пополнение числового поля $k$ по некоторой точке $\mathfrak{p}, \varepsilon_{n}$ - примитивный корень степени $n$ из единицы в алгебраическом замыкании некоторого поля нулевой характеристики, $n_{p}-p$-часть числа $n, n_{p^{\prime}}-p^{\prime}$-часть числа $n,(L: K)$ - размерность поля $L$ как векторного пространства над своим подполем $K$ (предполагается, что $L / K$ - конечное расширение), $\left[a_{1}, \ldots, a_{s}\right]$ - наименьшее общее кратное натуральных чисел $a_{1}, \ldots, a_{s}, \operatorname{Syl}_{p}(G)$ обозначает $p$-силовскую подгруппу абелевой группы $G, \bar{K}$ обозначает алгебраическое замыкание поля нулевой характеристики $K$.

2.2. Следующий результат является основным.

Теорема 3. Рассмотрим для конечного множества простых чисел $\pi$ класс $\mathfrak{G}_{\pi}$ конечных групп вида

$$
\mathfrak{G}_{\pi}:=\left\{G:|G|=\prod_{p \in \pi} p^{t_{p}}, t_{p} \in \mathbb{N}\right\} .
$$

Если $|\pi|>1$ либо $\pi=\{p \neq 2\}$, то оптимальной равномерной оценкой индекса Шура над полем $\mathbb{Q}$ неприводимьх характеров групп класса $\mathfrak{G}_{\pi}$ является

$$
m_{\mathbb{Q}}(\chi) \mid\left[\left\{(p-1)_{\pi}: p \in \pi, p \neq 2\right\}\right] \quad \forall G \in \mathfrak{G}_{\pi}, \quad \forall \chi \in \operatorname{Irr} G .
$$

Если же $\pi=\{2\}$, mо

$$
m_{\mathbb{Q}}(\chi) \mid 2 \quad \forall G \in \mathfrak{G}_{\pi}, \quad \forall \chi \in \operatorname{Irr} G .
$$

Дадим определение согласных круговых расширений числовых полей.

ОПРедЕлЕНиЕ 1. Пусть $k$ - числовое поле. Расширение $k\left(\varepsilon_{n}\right) / k$ называется согласным, если для любого простого делителя $p$ числа $n$ соответствующее силовское $p$-подрасширение $k\left(\varepsilon_{n}\right) / k_{p}$ согласно; $p$-расширение $k\left(\varepsilon_{n}\right) / k$ называется согласным, если для любой простой неархимедовой точки q поля $k$, не лежащей над $p$, пополненное расширение $k_{\mathfrak{q}}\left(\varepsilon_{n}\right) / k_{\mathfrak{q}}$ является циклическим. 
Класс универсально согласных $n$-круговых расширений числовых полей периода $m \mid n$, как легко следует из [4; теоремы 5,6 и комментарии к ним], содержится в классе согласных $n$-круговых расширений. Поэтому согласные расширения более приспособлены для теории индекса Шура. В этой теории важную роль играет теорема Голдшмидта-Айзекса (см. [5; теорема 1.3]). Из указанной теоремы и определения 1 непосредственно следует

ПРЕДЛОЖЕНИЕ 1. Согласность расширения числовых полей $k\left(\varepsilon_{n}\right) / k$ влечет за собой для произвольной конечной группы $G$ экспоненты (или порядка) $n u$ произвольного неприводимого характера $\chi \in \operatorname{Irr} G$ оценку $m_{k}(\chi) \mid 2$.

В связи с предложением 1, а также результатом [5; теорема 1.6] имеет смысл ответ на вопрос о существовании нециклических согласных круговых расширений числовых полей, а также установления критерия согласности. Вопросы такого рода изучаются в п. 3.4.

\section{§ 3. Доказательства теорем}

3.1. Дадим доказательство теоремы 1.

Рассмотрим конечную группу $G$ и неприводимый характер $\chi \in \operatorname{Irr} G$. Фиксируем произвольное простое число $p>2$ и докажем оценку

$$
m_{\mathbb{Q}_{p}}(\chi) \mid(p-1)
$$

Для доказательства оценки (3) достаточно доказать, что $m_{\mathbb{Q}_{p}(\chi)}(\chi)_{q} \mid(p-1)_{q}$ для любого простого $q$, делящего порядок $n$ группы $G$. Фиксируем произвольное простое $q \mid n$. Покажем, что

$$
m_{k_{q}}(\chi)_{q} \mid(p-1)_{q}
$$

где $k_{q}$ - поле, отвечающее $q$-силовской подгруппе в группе $\operatorname{Gal}\left(\mathbb{Q}_{p}\left(\varepsilon_{n}\right) / \mathbb{Q}_{p}(\chi)\right)$. Предположим сначала, что $q \neq p$. Если $k_{q}=\mathbb{Q}_{p}\left(\varepsilon_{n}\right)$, то результат (4) очевиден. В противном случае рассмотрим расширение $\mathbb{Q}_{p}\left(\varepsilon_{n}\right) / k_{q}\left(\varepsilon_{n_{p}}\right)$. Так как $\mathbb{Q}_{p}\left(\varepsilon_{n_{p}}\right) \subset k_{q}\left(\varepsilon_{n_{p}}\right)$, то в силу хорошо известных результатов о круговых расширениях расширение $\mathbb{Q}_{p}\left(\varepsilon_{n}\right) / k_{q}\left(\varepsilon_{n_{p}}\right)$ является неразветвленным.

Заметим теперь, что $k_{q}\left(\varepsilon_{n_{p}}\right)=k_{q}\left(\varepsilon_{p}\right)$ в силу нечетности числа $p$. В самом деле, группа $\operatorname{Gal}\left(k_{q}\left(\varepsilon_{n_{p}}\right) / k_{q}\left(\varepsilon_{p}\right)\right)$ канонически изоморфна группе $\operatorname{Gal}\left(\mathbb{Q}\left(\varepsilon_{n_{p}}\right) / K\right)$ для некоторого поля $K$. Ясно, что $\varepsilon_{p} \in K$, откуда следует равенство

$$
\left|\operatorname{Gal}\left(\mathbb{Q}\left(\varepsilon_{n_{p}}\right) / K\right)\right|_{q}=1
$$

Если доказать, что $m_{k_{q}\left(\varepsilon_{n_{p}}\right)}(\chi)=1$, то оценка $(4)$ в рассматриваемом нами случае $q \neq p$ будет получена.

Рассмотрим центрально-простую компоненту $A\left(k_{q}\left(\varepsilon_{n_{p}}\right), \chi\right)$ групповой алгебры $k_{q}\left(\varepsilon_{n_{p}}\right) G$. Как упоминалось в п. 1.1 индекс данной алгебры равен единице 
тогда и только тогда, когда расщепляется ей соответствующая циклотомическая алгебра $\left(\mathbb{Q}_{p}\left(\varepsilon_{n}\right) / k_{q}\left(\varepsilon_{n_{p}}\right), f\right)$. Построим расширение группы $\left\langle\varepsilon_{n}\right\rangle$ с помощью группы $\operatorname{Gal}\left(\mathbb{Q}_{p}\left(\varepsilon_{n}\right) / k_{q}\left(\varepsilon_{n_{p}}\right)\right)$ и соответствующего 2-коцикла $f$. Мы получим брауэровскую задачу погружения $\left(\mathbb{Q}_{p}\left(\varepsilon_{n}\right) / k_{q}\left(\varepsilon_{n_{p}}\right), H, \varphi,\left\langle\varepsilon_{n}\right\rangle\right)$, разрешимость которой эквивалентна расщеплению алгебры $A\left(k_{q}\left(\varepsilon_{n_{p}}\right), \chi\right)$. В [2; теорема 3.13.2] показано, что разрешимость данной задачи погружения равносильна тому, что образ класса $[f] \in H^{2}\left(\operatorname{Gal}\left(\mathbb{Q}_{p}\left(\varepsilon_{n}\right) / k_{q}\left(\varepsilon_{n_{p}}\right)\right),\left\langle\varepsilon_{n}\right\rangle\right)$ при подъеме в группу $H^{2}\left(\operatorname{Gal}\left(\overline{\mathbb{Q}}_{p} / k_{q}\left(\varepsilon_{n_{p}}\right)\right),\left\langle\varepsilon_{n}\right\rangle\right)$ является нулевым. Здесь, конечно, группа Галуа $\operatorname{Gal}\left(\overline{\mathbb{Q}}_{p} / k_{q}\left(\varepsilon_{n_{p}}\right)\right)$ действует на $\left\langle\varepsilon_{n}\right\rangle$ отступлением вдоль канонического эпиморфизма ограничения

$$
\psi: \operatorname{Gal}\left(\overline{\mathbb{Q}}_{p} / k_{q}\left(\varepsilon_{n_{p}}\right)\right) \rightarrow \operatorname{Gal}\left(\mathbb{Q}_{p}\left(\varepsilon_{n}\right) / k_{q}\left(\varepsilon_{n_{p}}\right)\right)
$$

Поскольку расширение $\mathbb{Q}_{p}\left(\varepsilon_{n}\right) / k_{q}\left(\varepsilon_{n_{p}}\right)$ является неразветвленным, то подъем класса $[f]$ можно осуществить сначала до группы $H^{2}\left(F_{1},\left\langle\varepsilon_{n}\right\rangle\right)$, где $F_{1}$ - группа Галуа максимального неразветвленного расширения поля $k_{q}\left(\varepsilon_{n_{p}}\right)$. Но хорошо известно, что группа Галуа максимального неразветвленного расширения локального поля является свободной проконечной группой. В частности, группа $H^{2}\left(F_{1},\left\langle\varepsilon_{n}\right\rangle\right)$ нулевая (см. [2; лемма 3.14$\left.]\right)$.

Итак, мы показали, что алгебра $A\left(k_{q}\left(\varepsilon_{n_{p}}\right), \chi\right)$ эквивалентна единице в группе Брауэра $B\left(k_{q}\left(\varepsilon_{n_{p}}\right)\right)$. Оценка (4) установлена для случая $q \neq p$.

Рассмотрим случай $q=p$. Нам достаточно показать согласно (4), что $m_{\mathbb{Q}_{p}}(\chi)_{p}=1$. Для этого воспользуемся [6; дополнение редактора, теорема 6]. В указанной теореме устанавливается, что $m_{\mathbb{Q}_{p}}(\chi)_{p}=1$ при $p>2$, и что $m_{\mathbb{Q}_{2}}(\chi)_{2} \mid 2$. При этом доказательство данной теоремы проще, чем доказательство исходной оценки.

Для случая $p=2$ нам остается показать, что $m_{\mathbb{Q}_{p}}(\chi)_{q}=1$ для произвольного нечетного простого $q$. Легко заметить, что если $k=\mathbb{Q}_{2}\left(\varepsilon_{n}\right)^{\operatorname{Syl}}{ }_{q}\left(\operatorname{Gal}\left(\mathbb{Q}_{2}\left(\varepsilon_{n}\right) / \mathbb{Q}_{2}\right)\right)$, то $\varepsilon_{n_{2}} \in k$. В частности, расширение $\mathbb{Q}_{2}\left(\varepsilon_{n}\right) / k$ неразветвлено. Теорема 1 полностью доказана.

3.2. Дадим доказательство теоремы 2 .

Фиксируем произвольную брауэровскую задачу $\left(k\left(\varepsilon_{n}\right) / K, H, \varphi,\left\langle\varepsilon_{m}\right\rangle\right)$, где расширение $k\left(\varepsilon_{n}\right) / K$ является подрасширением в $k\left(\varepsilon_{n}\right) / k$, которое удовлетворяет условиям теоремы 2 , а $m \mid n$.

Лемма 1. Задача $\left(k\left(\varepsilon_{n}\right) / K, H, \varphi,\left\langle\varepsilon_{m}\right\rangle\right)$ разрешима.

Доказательство. В самом деле, задаче $\left(k\left(\varepsilon_{n}\right) / K, H, \varphi\right)$ соответствует в смысле п. 1.1 циклотомическая алгебра $\left(k\left(\varepsilon_{n}\right) / K, f\right), 2$-коцикл $f$ которой соответствует 2-коциклу расширения

$$
1 \longrightarrow\left\langle\varepsilon_{m}\right\rangle \longrightarrow H \stackrel{\varphi}{\longrightarrow} \operatorname{Gal}\left(k\left(\varepsilon_{n}\right) / K\right) \longrightarrow 1 \text {. }
$$

Хорошо известно (см. [1; предложение 2.1]), что всякая циклотомическая алгебра над полем $K$ является простой компонентой $K$-групповой алгебры некоторой конечной группы. Поэтому если $k\left(\varepsilon_{n}\right) / K$ не является 2-расширением, то 
результат немедленно следует из теоремы 1. В противном случае из условия теоремы 2 следует (см. [1; теорема 5.3]), что подгруппа Шура $S(K)$ группы Брауэра $B(K)$ тривиальна, что доказывает лемму и в этом случае.

Возвращаясь к доказательству теоремы 2, отметим, что достаточно установить выполнение условия согласности для произвольной задачи погружения расширения $k\left(\varepsilon_{n}\right) / k$ с ядром периода $n$. Из п. 1.1 следует, что достаточно доказать разрешимость произвольной брауэровской задачи погружения для произвольного подрасширения $k\left(\varepsilon_{n}\right) / K$ расширения $k\left(\varepsilon_{n}\right) / k$ с циклическим ядром порядка $m \mid n$. Применим лемму 1 и получим искомый результат.

3.3. Установим основную теорему 3. Прежде всего отметим, что оценка (2) действительно имеет место согласно [1].

Предположим противное. Тогда множество $\pi$ состоит более чем из одного элемента (иначе результат следует из [5; теорема 1.3]). По предположению найдется простое число $q \in \pi$ такое, что $q$-часть оценки из (2) улучшаема равномерно на классе $\mathfrak{G}_{\pi}$. Это означает, что найдется нечетное простое число $p \in \pi$, удовлетворяющее условию

$$
(p-1)_{q}=\left[\left\{(r-1)_{\pi}: r \in \pi, r \neq 2\right\}\right]_{q} .
$$

Заметим, что если удастся построить конечную $\{p, q\}$-группу $H$ и неприводимый характер $\chi \in \operatorname{Irr} H$ такой, что $m_{\mathbb{Q}}(\chi)=(p-1)_{q}$, то $q$-часть оценки $(2)$ улучшить нельзя вопреки предположению. В самом деле, рассмотрим произвольную конечную группу $H_{1}$ класса $\mathfrak{G}_{\pi \backslash\{p, q\}}$ и ее главный характер $1_{H_{1}}$. Неприводимый характер $\chi \otimes 1_{H_{1}}$ группы $H \times H_{1}$ будет иметь индекс Шура над полем рациональных чисел, равный $(p-1)_{q}$. Полученное противоречие будет в силу произвольности выбора $q$ означать неулучшаемость оценки (2).

Лемма 2. Существует $\{p, q\}$-группа $H$ и неприводимый характер $\chi \in \operatorname{Irr} H$ maкue, чmо $m_{\mathbb{Q}}(\chi)=(p-1)_{q}$.

ДоказАТЕЛЬСтво. Известно (см. [1; теоремы 4.4, 6.1]), что для $\{p, q\}$-групп, где $q \mid(p-1)$ и $q \neq 2$, справедлива оценка

$$
m_{\mathbb{Q}}(\chi) \mid\left(\frac{p-1}{e}, q^{\lambda}\right),
$$

где $e$ - индекс слабого ветвления некоторой (произвольной) простой точки $\mathfrak{p}$ поля $\mathbb{Q}(\chi)$, лежащей над $p$, а $q^{\lambda}$ - максимальная степень $q$-примарного примитивного корня из единицы, содержащегося в поле $\mathbb{Q}(\chi)$. В случае $q=2$ оценка $(6)$ будет справедливой при $(p-1) / e \equiv 0(\bmod 2)$. В противном случае оценку (6) надо еще умножить на 2.

Рассмотрим число $n=p q^{\beta_{0}}$, где натуральное число $\beta_{0}$ выбрано так, чтобы $q^{\beta_{0}} \geqslant(p-1)_{q}$. Положим

$$
Z:=\mathbb{Q}\left(\varepsilon_{p q^{\beta_{0}}}\right)^{\operatorname{Syl}_{q}\left(\operatorname{Gal}\left(\mathbb{Q}\left(\varepsilon_{p q^{\beta_{0}}}\right) / \mathbb{Q}\left(\varepsilon_{(p-1)_{q}}\right)\right)\right)} .
$$


В [7; доказательство теоремы 1] показано, что существуют натуральное число $\beta \geqslant \beta_{0}$, конечная группа $H$ порядка $p q^{\beta}$ и неприводимый характер $\chi \in \operatorname{Irr} H$ такие, что

$$
Z=\mathbb{Q}(\chi), \quad m_{Z}(\chi)=\left(\frac{p-1}{e}, q^{\lambda}\right)
$$

где параметры $\lambda$, е соответствуют параметрам из (6). Но поле $Z$ было выбрано так, чтобы

$$
\left(\frac{p-1}{e}, q^{\lambda}\right)=\left(\frac{p-1}{e}\right)_{q} .
$$

Более того, $e_{q}=1$. В самом деле, точка $p$ поля $\mathbb{Q}$ вполне распадается в поле $\mathbb{Q}\left(\varepsilon_{(p-1)_{q}}\right)$, а $q \nmid\left(Z: \mathbb{Q}\left(\varepsilon_{(p-1)_{q}}\right)\right)$. Заметим, что если $q \neq 2$, то доказательство теоремы 1 из [7] приложимо непосредственно. Если же $q=2$ и $4 \mid(p-1)$, то рассуждения проходят дословно. В случае $q=2$ и $4 \nmid(p-1)$ просто рассмотрим группу кватернионов $Q_{8}$, у которой существует согласно [5; лемма 2.1] неприводимый характер $\chi$ такой, что $m_{\mathbb{Q}}(\chi)=2$. Затем возьмем произвольную конечную $p$-группу $H_{1}$, ее главный характер $1_{H_{1}}$. Конечная группа $Q_{8} \times H_{1}$ с характером $\chi \otimes 1_{H_{1}}$ будет в этом случае искомой. Лемма доказана.

Из леммы 2 и приводимых выше в настоящем пункте рассуждений следует результат теоремы 3 .

3.4. Критерий согласности расширения. Рассмотрим нетривиальное $p$-расширение $\mathbb{Q}\left(\varepsilon_{n}\right) / k$, где $p \mid n$. Выберем произвольным образом циклическую подгруппу $M$ группы $\operatorname{Gal}\left(\mathbb{Q}\left(\varepsilon_{n}\right) / k\right)$. Положим $K:=\mathbb{Q}\left(\varepsilon_{n}\right)^{M}$. Так как группа $M$ является циклической $p$-группой, то решетка подполей в расширении $\mathbb{Q}\left(\varepsilon_{n}\right) / K$ есть линейно упорядоченное по включению множество. Можно поэтому считать, что

$$
K \subseteq K\left(\varepsilon_{p_{1}^{k_{1}}}\right) \subseteq K\left(\varepsilon_{p_{2}^{k_{2}}}\right) \subseteq \cdots \subseteq K\left(\varepsilon_{p_{s}^{k_{s}}}\right) \subseteq K\left(\varepsilon_{n}\right)=\mathbb{Q}\left(\varepsilon_{n}\right),
$$

где $n=p_{1}^{k 1} \cdots p_{s}^{k_{s}}-$ каноническое разложение на простые множители. Докажем, что на самом деле $K\left(\varepsilon_{p_{s}^{k_{s}}}\right)=K\left(\varepsilon_{n}\right)$. Рассмотрим произведение $\varepsilon_{p_{1}^{k_{1}}} \cdots \varepsilon_{p_{s}^{k_{s}}}$ и заметим, что

$$
\varepsilon_{p_{j}^{k_{j}}}=\varepsilon_{n}^{n_{p_{j}^{\prime}}}=\exp \left\{\frac{2 \pi i}{p_{j}^{k_{j}}}\right\}
$$

поэтому

$$
\varepsilon_{p_{1}^{k_{1}}} \cdots \varepsilon_{p_{s}^{k_{s}}}=\exp \left\{2 \pi i \frac{\sum_{j=1}^{s} p_{1}^{k_{1}} \cdots p_{j-1}^{k_{j-1}} p_{j+1}^{k_{j+1}} \cdots p_{s}^{k_{s}}}{n}\right\} .
$$

Но число $\sum_{j=1}^{s} p_{1}^{k_{1}} \cdots p_{j-1}^{k_{j-1}} p_{j+1}^{k_{j+1}} \cdots p_{s}^{k_{s}}$ взаимно просто с $n$. Это означает, что поле $K\left(\varepsilon_{p_{s}^{k}}\right)$ содержит некоторый примитивный корень степени $n$ из единицы. В силу нормальности расширения $K\left(\varepsilon_{p_{s}^{k_{s}}}\right) / K$ заключаем, что $K\left(\varepsilon_{p_{s}^{k_{s}}}\right)=$ $K\left(\varepsilon_{n}\right)=\mathbb{Q}\left(\varepsilon_{n}\right)$. Обозначим $r:=p_{s}$. Легко видеть, что расширение $\mathbb{Q}\left(\varepsilon_{n}\right) / K=$ $K\left(\varepsilon_{n_{r}}\right) / K$ неразветвлено по всем неархимедовым простым точкам поля $K$, не лежащим над $r$. 
Предположим, что расширение $\mathbb{Q}\left(\varepsilon_{n}\right) / k$ является согласным. Заметим, что если $n=p^{\alpha}$, то расширение $\mathbb{Q}\left(\varepsilon_{n}\right) / k$ согласно по определению. Будем считать, что $n$ не является степенью числа $p$. Возьмем тогда (если существует) произвольное нечетное простое $q \mid n$ такое, что $q \neq p$. Если $p \nmid(q-1)$, то $\varepsilon_{n_{q}} \in k$. Поэтому в этом случае для произвольной простой точки q поля $k$, лежащей над $q, \mathbb{Q}_{q}\left(\varepsilon_{n}\right)=K_{\mathfrak{q}}\left(\varepsilon_{n_{q^{\prime}}}\right)$, в силу чего расширение $\mathbb{Q}\left(\varepsilon_{n}\right) / k$ неразветвлено по $\mathfrak{q}$, а значит, является циклическим. Это означает, что мы можем ограничиться лишь такими нечетными простыми $q \mid n$, которые удовлетворяют условию $p \mid(q-1)$.

Фиксируем произвольную простую точку q поля $k$, лежащую над выбранным числом $q$. По предположению расширение $\mathbb{Q}_{q}\left(\varepsilon_{n}\right) / k_{\mathfrak{q}}$ является циклическим. Если при этом оно не является универсально согласным периода $p$, то согласно $\left[4\right.$; теорема 6] индекс ветвления расширения $\mathbb{Q}_{q}\left(\varepsilon_{n}\right) / k_{\mathfrak{q}}$ не меньше числа $\left(q^{f}-1\right)_{p}$, где $f$ - степень инерции расширения $k_{\mathfrak{q}} / \mathbb{Q}_{q}$. С другой стороны, ветвление расширения $\mathbb{Q}_{q}\left(\varepsilon_{n}\right) / k_{\mathfrak{q}}$ слабое, так как $q \neq p$. Но тогда индекс ветвления расширения $\mathbb{Q}_{q}\left(\varepsilon_{n}\right) / k_{\mathfrak{q}}$ в точности равен $\left(q^{f}-1\right)_{p}$. Поскольку число $\left(q^{f}-1\right)_{p}$ должно делить индекс ветвления расширения $\mathbb{Q}_{q}\left(\varepsilon_{n}\right) / \mathbb{Q}_{q}$, равный $\varphi\left(n_{q}\right)$ ( $\varphi$ обозначает функцию Эйлера), то $\left(q^{f}-1\right)_{p} \mid \varphi\left(n_{q}\right)_{p}$, откуда

$$
\left(q^{f}-1\right)_{p}=(q-1)_{p}
$$

В частности, равенство $(7)$ означает, что расширение $k_{\mathfrak{q}}\left(\varepsilon_{q}\right) / k_{\mathfrak{q}}$ вполне разветвлено с максимально возможным ветвлением.

Итак, если расширение $\mathbb{Q}\left(\varepsilon_{n}\right) / k$ согласно, то для всякого (если такое существует) нечетного простого $q \mid n$ с условием $p \mid(q-1)$ расширение $\mathbb{Q}_{q}\left(\varepsilon_{n}\right) / k_{\mathfrak{q}}$ либо является универсально согласным периода $p$, либо расширение $k_{\mathfrak{q}}\left(\varepsilon_{q}\right) / k_{\mathfrak{q}}$ вполне разветвлено с индексом ветвления, равным $(q-1)_{p}$. В последнем случае расширение $\mathbb{Q}_{q}\left(\varepsilon_{n}\right) / k_{\mathfrak{q}}$ будет циклическим, лишь когда $\mathbb{Q}_{q}\left(\varepsilon_{n}\right)=k_{\mathfrak{q}}\left(\varepsilon_{q}\right)$. В самом деле, из условия цикличности и рассуждений в начале п. 3.4 вытекает, что необходимо $\mathbb{Q}_{q}\left(\varepsilon_{n}\right)=k_{\mathfrak{q}}\left(\varepsilon_{n_{p_{s}}}\right)$ для некоторого простого $p_{s} \mid n$. Если $p_{s} \neq q$, то расширение $\mathbb{Q}_{q}\left(\varepsilon_{n}\right) / k_{\mathfrak{q}}$ будет неразветвленным вопреки предположению. Поэтому $p_{s}=q$, в частности, $\mathbb{Q}_{q}\left(\varepsilon_{n}\right)=k_{\mathfrak{q}}\left(\varepsilon_{q}\right)$. Но тогда группа $\operatorname{Gal}\left(\mathbb{Q}_{q}\left(\varepsilon_{n}\right) / k_{\mathfrak{q}}\right)$ является с точностью до изоморфизма подгруппой в мультипликативной группе поля $\mathbb{F}_{q^{f}}$, а значит, циклической.

ПРЕДЛОжЕНИЕ 2. p-расширение $\mathbb{Q}\left(\varepsilon_{n}\right) / k$ согласно тогда и толъко только тогда, когда для любого нечетного простого $q \mid n$ такого, что $q \neq p, p \mid(q-1)$, либо $\mathbb{Q}_{q}\left(\varepsilon_{n}\right) / k_{\mathfrak{q}}$ - универсально согласное расширение периода $p$, либо $\varepsilon_{n_{q^{\prime}}} \in k_{\mathfrak{q}}$. Здесъ q - произвольная точка поля $k$, лежсащая над $q$.

ДокАзАтельство. Необходимость нами уже установлена. Достаточность очевидным образом следует из [4; теорема 6], неразветвленности расширения $k_{\mathfrak{q}}\left(\varepsilon_{n_{q^{\prime}}}\right) / k_{\mathfrak{q}}$ и цикличности группы $\operatorname{Gal}\left(k_{\mathfrak{q}}\left(\varepsilon_{q}\right) / k_{\mathfrak{q}}\right)$. Заметим, что при $p=2$ согласно определению 1 анализировать 2-адическое пополнение расширения $\mathbb{Q}\left(\varepsilon_{n}\right) / k$ не требуется. При $p>2$-адическое пополнение расширения $\mathbb{Q}\left(\varepsilon_{n}\right) / k$ заведомо будет неразветвленным, а значит, циклическим. 
Предложение 2 показывает, что, вообще говоря, оценку в виде наибольшего общего делителя оценки (2) и оценки из [5; теорема 1.6] трудно улучшить указанным методом, зная лишь порядок группы. Тем не менее, приведем пример, когда такое улучшение возможно. Данный пример особенно показателен в контексте теоремы 3 .

ПримеР. Рассмотрим произвольную конечную группу $G$, порядок которой имеет вид $n=p^{\alpha} q^{\beta}$, где $2<p<q, p^{\alpha} \mid(q-1), \alpha \geqslant 2$. Применение (2) дает оценку $m_{\mathbb{Q}}(\chi) \mid p^{\alpha}$ для произвольного неприводимого характера $\chi \in \operatorname{Irr} G$. Применение оценки из [5; теорема 1.6] дает $m_{\mathbb{Q}}(\chi) \mid p^{\alpha-1}$.

Рассмотрим цепочку включений

$$
\mathbb{Q} \subset k \subset \mathbb{Q}\left(\varepsilon_{n}\right),
$$

где $k=\mathbb{Q}\left(\varepsilon_{n}\right)^{\operatorname{Syl}_{p}\left(\operatorname{Gal}\left(\mathbb{Q}\left(\varepsilon_{n}\right) / \mathbb{Q}\right)\right)}$. Легко видеть, что $\varepsilon_{n_{p}} \in k_{\mathfrak{q}}$ для произвольной простой точки q поля $k$, лежащей над $q$. Действительно, в силу $p^{\alpha} \mid(q-1)$ имеем даже $\varepsilon_{n_{p}} \in \mathbb{Q}_{q}$. Наконец, заметим нецикличность группы $\operatorname{Gal}\left(\mathbb{Q}\left(\varepsilon_{n}\right) / k\right)$.

Мы получили пример согласного нециклического расширения $\mathbb{Q}\left(\varepsilon_{n}\right) / k(\mathrm{~cm}$. предложение 2). Как следствие для любого неприводимого характера $\chi \in \operatorname{Irr} G$ имеем $m_{\mathbb{Q}}(\chi)=1$.

Резюмируя, стоит обратить внимание на тот факт, что оценка индекса Шура неприводимого комплексного характера $\chi$ конечной группы $G$ порядка $n$ над полем рациональных чисел, основанная на использовании максимального по порядку согласного подрасширения в расширении $\mathbb{Q}\left(\varepsilon_{n}\right) / \mathbb{Q}$, является конструктивной. В самом деле, если $K$ - подполе поля $\mathbb{Q}\left(\varepsilon_{n}\right)$ такое, что расширение $\mathbb{Q}\left(\varepsilon_{n}\right) / K$ согласно, то представление с характером $\chi$ реализуется в поле $K(\chi)$ или в поле $K(\chi, \sqrt{-1})$. В частности, оценка теоремы 1.6 статьи [5] конструктивна. Оценка (2) неконструктивна в этом смысле.

Автор считает своим приятным долгом выразить благодарность А. В. Яковлеву за чрезвычайно полезные обсуждения вопросов теории погружения.

\section{Список литературы}

[1] T. Yamada, The Schur subgroup of the Brauer group, Lecture Notes in Math., 397, Springer-Verlag, Berlin-New York, 1974.

[2] В. В. Ишханов, Б. Б. Лурье, Д. К. Фаддеев, Задача погружения в теории Галуа, Наука, M., 1990; англ. пер.: V. V. Ishkhanov, B. B. Lur'e, D. K. Faddeev, The embedding problem in Galois theory, Transl. Math. Monogr., 165, Amer. Math. Soc., Providence, RI, 1997.

[3] А. В. Яковлев, “Задача погружения полей”, Изв. АН СССР. Сер. матем., 28:3 (1964), 645-660.

[4] Н. П. Зяпков, А. В. Яковлев, "Универсально согласные расширения Галуа", Модули и представления, Зап. научн. сем. ЛОМИ, 71, Изд-во "Наука", Ленинград. отд., Л., 1977, 133-152; англ. пер.: N. P. Zyapkov, A. V. Yakovlev, "Universally concordant Galois extensions", J. Soviet Math., 20:6 (1982), 2595-2609.

[5] Д. Д. Киселев, "Поля разложения конечных групп", Изв. РАН. Сер. матем., 76:6 (2012), 95-106; англ. пер.: D. D. Kiselev, "Splitting fields of finite groups", Izv. Math., 76:6 (2012), 1163-1174. 
[6] Ч. Кэртис, И. Райнер, Теория представлений конечных групп и ассоциативных алгебр, Наука, М., 1969; пер. с англ.: Ch. W. Curtis, I. Reiner, Representation theory of finite groups and associative algebras, Wiley, New York-London, 1962.

[7] Ю. Л. Баранник, "О телах, возникающих в разложении рациональных групповых алгебр конечных групп порядка $p^{\alpha} q^{\beta}$, Изв. вузов. Матем., 1977, № 12, 13-18.

\section{Д. Д. Киселев (D. D. Kiselev)}

Механико-математический факультет

Московского государственного университета

им. М. В. Ломоносова

E-mail: denmexmath@yandex.ru
Поступила в редакцию 12.09 .2012 и 25.12 .2012 